Cahiers de philosophie de l'université de

\title{
Autour de la totalité (et du non-être) dans la Métaphysique d'Avicenne
}

\section{Olga L. Lizzini}

\section{(2) OpenEdition}

1 Journals

Édition électronique

URL : https://journals.openedition.org/cpuc/339

DOI : $10.4000 /$ cpuc.339

ISSN : 2677-6529

Éditeur

Presses universitaires de Caen

\section{Édition imprimée}

Date de publication : 31 décembre 2016

Pagination : 55-76

ISBN : 978-2-84133-842-9

ISSN : 1282-6545

\section{Référence électronique}

Olga L. Lizzini, « Autour de la totalité (et du non-être) dans la Métaphysique d'Avicenne », Cahiers de philosophie de l'université de Caen [En ligne], 53 | 2016, mis en ligne le 01 février 2019, consulté le 31 janvier 2023. URL : http://journals.openedition.org/cpuc/339; DOI : https://doi.org/10.4000/cpuc.339

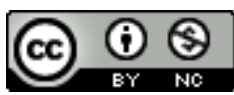

Creative Commons - Attribution - Pas d'Utilisation Commerciale 4.0 International - CC BY-NC 4.0 https://creativecommons.org/licenses/by-nc/4.0/ 


\section{Autour de la totalité (et du non-être) dans la Métaphysique d'Avicenne}

\section{Les termes}

L'idée de totalité se trouve dans la Métaphysique du Livre de la Guérison (les Ilāhiyyāt du Kitāb al-Šifáa $)^{1}$ d'Avicenne en relation avec plusieurs thèmes. Sans prétendre être exhaustif, on peut tenter d'en dresser une liste.

Commençons par mentionner le thème de la totalité absolue de l'être, voire de l'existence, qui, pour Avicenne, est de fait divisée en parties: celle qui relève de l'existence non causée et celle qui relève de l'existence causée. L'existence du Principe, en soi absolument nécessaire, s'oppose à l'existence du monde, qui est absolument créé, voire rendu nécessaire dans son existence. Les deux constituent (ou devraient constituer) ensemble la totalité de l'être. À la position de l'être dans sa totalité, il faut en effet chez Avicenne attacher immédiatement une distinction: le Principe n'est principe que d'une partie de l'être, précisément de celle qui correspond à l'être qui est causé ou rendu nécessaire ${ }^{2}$. Cela signifie aussi - on le verra - qu'une conception précise de la création doit être élaborée: la séparation entre ce qui est non causé et ce qui est causé exige une pensée de la création où le tout de l'être causé soit posé dans l'existence. La conception de la totalité devient alors - et on repère ici un deuxième thème pour la pensée de la totalité - la clé même pour expliquer la création. Mais - il faut le préciser -

1. Ilāhiyyāt: Ibn Sīnā, K. al-Šifä'. Al-Ilāhiyyāt (Al-Shifä’. La Métaphysique), t. I, traités I-V, G. C. Anawati et S. Zayed (éd.), t. II, traités VI-X, M. Y. Mousa, S. Dunya et S. Zayed (éd.), Le Caire, Ministère de la Culture et de l'Orientation, 1960. Je tiens à remercier Maud Pouradier pour ses suggestions, qui m’ont permis d'améliorer le style de mon article.

2. Si le Principe fait partie de l'être, il ne peut être le principe que d'une partie de l'être. Si le Principe pouvait être principe de tout l'être, il devrait - et c'est un paradoxe - être principe de soi-même. Le tout en tant que tel n'a pas de principe: Ilāh., I, 2, 14, 1-16, 20. 
la distinction ou l'articulation de la totalité de l'être que l'on vient d'évoquer a d'autres conséquences, notamment sur le plan théorétique.

Tout d'abord, la modulation de ce qu'on définit parfois comme la théorie avicennienne de l'univocité de l'être: si l'être ou - dans les termes plus proprement avicenniens - l'existence (al-wuğūd) est une totalité, le problème est en effet celui de comprendre dans quels termes il faut moduler la distinction de ses parties, celle qui relève de l'être du Principe et celle qui relève de l'être du monde.

En ce sens, la conception de l'être en tant que totalité montre que l'univocité de l'être ne peut pas être conçue en dehors de la hiérarchie et donc, in fine, de l'analogie de l'être: l'existence en soi nécessaire du Principe est supérieure à celle du monde, laquelle est nécessaire par un autre ${ }^{3}$.

À ces trois premiers thèmes, un quatrième pourrait être ajouté. Avicenne parle en effet du «tout», ou littéralement de «l'ensemble» (ğumla: «une totalité d'unités »), en discutant de la chaîne causale qui est telle seulement si elle est complète, et donc finie, en ce qui concerne tous ses éléments: les causes intermédiaires (qui sont aussi des causés) et le dernier effet (ce qui est causé de façon absolue et dont rien ne dérive) renvoient nécessairement à une cause première et absolue ${ }^{4}$.

On pourrait mentionner encore un cinquième thème, celui de l'universalité: l'universel (al-kullī) est l'intention (al-ma'nā) où la totalité des possibilités de la chose se retrouvent en tant que prédicables 5 .

On reconnaît tous ces thèmes dans différents termes utilisés par Avicenne: ğumla, kull, tāmm. Si l'on en reste au terme qui signifie la totalité à la lettre - al-kulliyya -, c'est - comme on le verra - le sens d'absolu de

3. Sur la question de l'univocité et de l'analogie de l'être chez Avicenne, T. Koutzarova, Das Transzendentale bei Ibn Sīnā. Zur Metaphysik als Wissenschaft erster Begriffs- und Urteilsprinzipien, Leyde - Boston, Brill, 2009; A. Treiger, «Avicenna's Notion of Transcendental Modulation of Existence (taškīk al-wuğùd) and its Greek and Arabic Sources», in Islamic Philosophy, Science, Culture, and Religion. Studies in Honor of Dimitri Gutas, F. Opwis et D. C. Reisman (dir.), Leyde, Brill, 2014; S. Menn, «Avicenna’s Metaphysics», in Interpreting Avicenna. Critical Essays, P. Adamson (dir.), Cambridge, Cambridge University Press, 2013, p. 143-169; T.-A. Druart, «Ibn Sina and the Ambiguity of Being's Univocity», in Views on the Philosophy of Ibn Sina \& Mulla Sadra Shirazi, M. A. Mensia (dir.), Carthage, The Tunisian Academy of Sciences, Letters and Arts Beit al-Hikma, 2014, p. 15-24; D. De Haan, "The Doctrine of the Analogy of Being in Avicenna's Metaphysics of the Healing", The Review of Metaphysics, ${ }^{\circ}$ 69, 2015, p. 261-286.

4. À propos de l'analyse de la totalité (ğumla) des effets dans ce contexte, J. McGinnis, Avicenna, Oxford, Oxford University Press, 2010, p. 164-168.

5. Ilāh., V, 1, en particulier, p. 195, 6-15. Plus précisément, la formulation de la possibilité est ici celle de la non-impossibilité, ou encore plus exactement de l'impossibilité d'un empêchement de la prédication multiple. A. de Libera, L'Art des généralités. Théories de l'abstraction, Paris, Aubier, 1999, p. 499-607; cf. T. A. Druart, «Avicennan Troubles: the Mysteries of the Heptagonal House and of the Phoenix ", Tópicos, nº 42, 2012, p. 51-74. 
la création, voire de l'instauration ( $a l-i b d \bar{a})$, qui se présente au premier plan. Toutes les distinctions que l'on a rappelées dérivent du vocabulaire originaire de la métaphysique avicennienne. Toutefois, afin d'analyser la contribution possible d'Avicenne à l'histoire du concept de totalitas, il semble qu'un changement de perspective soit nécessaire. Autrement dit, c'est l'Avicenna Latinus qu'il faut regarder ${ }^{6}$. Comme tous les penseurs que le Moyen Âge connut grâce aux traductions, Avicenne / Ibn Sīnā est en fait l'auteur de textes que l'on pourrait définir comme doubles, l'original arabe étant comme redoublé par sa version latine. Les correspondances terminologiques sont parfois imparfaites, voire fautives, mais elles sont aussi parfois le signe d'une vraie compréhension; plus que cela, les termes originaux peuvent être occasionnellement éclairés, voire accentués par la traduction ${ }^{7}$.

Le cas de totalitas - abstrait tardif de totus, tota, totum ${ }^{8}$ - est en ce sens exemplaire. À en examiner les occurrences (peu nombreuses à vrai dire) dans le Liber de philosophia prima (dans le Liber de anima, on n'enregistre aucune occurrence), on trouve six passages qui témoignent de l'usage du terme. Un premier passage concerne la question des transformations naturelles et donc de la réception de la forme de la part de la matière; totalitas y traduit l'arabe mağmū', que l'on pourrait rendre, plutôt que par l'idée de totalité, par l'idée d'ensemble ou de ce qui se réunit. C'est en effet de l'ensemble de la matière et de la forme qu'Avicenne discute ici ${ }^{9}$.

On trouve ensuite trois passages où le terme correspond à ğumla:

1. Il s'agit dans le premier cas d'un passage qui discute de la définition de l'unité du continu (toute la section est consacrée à la définition de l'un) : l'ensemble des lignes - ou tout simplement les deux lignes - qui se croisent sur un angle constituent une (imparfaite) unité ${ }^{10}$.

6. Liber de philosophia prima sive scientia divina, t. I [livres I-IV], t. II [livres V-X ] et t. III [Lexiques], S. van Riet (éd.), Louvain-la-Neuve - Leyde, Peeters - Brill, 1977, 1980, 1983.

7. Pour l'analyse des termes de l'être et de la création en ce sens, J. Jolivet, Perspectives médiévales et arabes, Paris, Vrin, 2006, p. 217-227.

8. Dans A. Ernout et A. Meillet, Dictionnaire étymologique de la langue latine. Histoire des mots, 4 e éd., Paris, Klincksieck, 1959, p. 1232-1233, seuls totus, tota et totum sont enregistrés.

9. «Quia igitur secunda pars divisionis est quod substantia prioris fit in eo quod est posterius quasi ipsum idem vel pars eius, et prima est quod totalitas [mağmū] substantiae primi et perfectio adiungatur illi, et fuit etiam, sicut iam nosti ex praedictis, ut id quod est finitum in effectu, non habens partes infinitas in effectu, sive sint partes mensurabiles sive intelligibiles ita ut habeant ordinem, tunc hoc sufficit nobis ne opus sit ostendere an sit possibile esse subiectum huiusmodi ante subiectum usque infinitum, an non sit possibile», in Liber de philosophia prima, VIII, 1, p. 380, 95-3. La traduction latine ne respecte pas complètement le texte arabe, mais le sens général est maintenu: Ilāh., VIII, 1, p. 330, 9-13.

10. "Sed verum unum est id in quo est multitudo in potentia tantum, ut in lineis, illa scilicet in qua non est angulus, et in superficiebus, illa quae est planissima, et in corporibus, illud quod circumdat superficies in qua non est curvatura secundum angulum. Post hoc autem 
2. Dans le deuxième cas, l'idée de totalité, qui est encore une fois plus littéralement celle de l'ensemble (ğumla), sert - dans un passage très compliqué - à définir la cause de ce qui - tout en étant possible - vient à l'être ${ }^{11}$. Le sens du passage peut être résumé de la sorte: la possibilité est pour Avicenne une disposition neutre. Elle est identique par rapport à l'existence comme à la non-existence, de sorte que la véritable cause de ce qui fait passer le possible à l'existence est dans un ensemble d'éléments : un ensemble formé, d'un côté, par la cause efficiente et, de l'autre, par la raison (ou les raisons) qui rendent l'existence et donc l'action de la même cause efficiente prépondérantes. C'est en ce sens la prépondérance qui légitime la cause dans son action de faire être: dans le cas de la nature et de la matière, la prépondérance est due à la préparation appropriée ${ }^{12}$.

3. Le troisième exemple de totalitas (traduisant encore une fois ğumla) se trouve enfin dans le contexte d'un discours cosmologique: Avicenne l'utilise pour affirmer que le corps de la dernière sphère est contenu ou compris par l'ensemble ou la totalité de la sphère dernière ${ }^{13}$. Dans toutes

sequitur aliud unum in quo est multitudo in effectu, sed duo eius extrema copulantur apud terminum communem, sicut totalitas [ğumla] duarum linearum continentium angulum ", in Liber de philosophia prima, III, 2, p. 109, 10-16: cf. Iläh., III, 2, p. 98, 16-99, 1.

11. «Igitur possibilitas rem essendi per causam, quantum ad ipsam essendum per illam et non essendum per illam, una est; et cuius comparatio fuerit ad essendum rem per illam et non essendum rem per illam, una, ipsam esse causam non est dignius quam non esse causam. Sed certus intellectus facit debere hic esse dispositionem qua discernatur suum esse per illam a suo non esse per illam. Si autem fuerit illa dispositio etiam quae facit debere esse hanc discretionem, et haec dispositio fuerit attributa causae et habuerit esse, tunc totalitas [ğumla] essentiae et eius quod adiungitur ei erit ipsa causa; ante hoc autem, essentia erat subiectum causalitatis et erat talis quod posset vere fieri causa", in Liber de philosophia prima, IV, 1, p. 188, 83, p. 189, 93; cf. Iläh., IV, 1, p. 166, 4-12.

12. Dans le cas du possible, le passage à l'existence est concevable a posteriori. J'ai analysé ce point dans Fluxus (fayd). Indagine sui fondamenti della metafisica e della fisica di Avicenna, Bari, Edizioni di Pagina, 2011, p. 166-167, 231-233, 310-311, 336-337 et 431-434 ; je renvoie également à mon article " "A Mysterious Order of Possibles". Some Remarks on the Views of Avicenna and Aquinas on Creation: al-Ilāhiyyāt, the Quaestiones De potentia and Beatrice Zedler's Interpretation", American Catholic Philosophical Quarterly, n 88, 2014, p. 237-270; plus spécifiquement sur la doctrine de la préparation de la matière chez Avicenne et ses sources, Ibrahim Halil Üçer, «Aristotle’s Dunamis Transformed: On Avicenna’s Conception of Natural Isti'dād and Tahayyu'», Nazariyat. Journal for the History of Islamic Philosophy and Sciences, $\mathrm{n}^{\circ}$ 2/3, 2015, p. 37-76.

13. "Igitur ex prima intelligentia, inquantum intelligit primum, sequitur esse alterius intelligentiae inferioris ea, et inquantum intelligit seipsam, sequitur ex ea forma caeli ultimi et eius perfectio et haec est anima, et propter naturam essendi possibile quae est ei et quae est retenta inquantum intelligit seipsam, est esse corporeitatis caeli ultimi quae est contenta in totalitate [ğumla] caeli ultimi», in Liber de philosophia prima, IX, 4, p. 483, 85-91; cf. Iläh., IX, 4, p. 406, 16-19. Sur les questions cosmologiques impliquées par ce passage, D. Janos, «Moving the Orbs: Astronomy, Physics, and Metaphysics and the Problem of Celestial Motion according to Ibn Sīnā », Arabic Sciences and Philosophy, no 21 (o2), 2011, p. 165-214. 
ces occurrences, l'idée principale est également celle d'ensemble ou d'entier; le terme arabe ğumla correspond certainement à l'idée de totalité, mais toujours en vertu du sens sous-entendu de «somme» ou d'«ensemble» ou «grande quantité».

Dans d'autres contextes, le terme est traduit par collectio, compositio, coniunctio et universitas (pour mağmū' on enregistre également: collectio, compositio ou bien aggregatio, coniunctio) ${ }^{14}$. Dans deux passages, le terme totalitas représente enfin kulliyya, l'abstrait de l'arabe kull dont la traduction est simplement «tout»: al-kull, c'est «le tout». Lorsqu'il précède un nom, le terme kull donne la même idée que le français «tout» ou - selon le cas - que le français "chaque» (kull insān, c'est bien «chaque homme» ou «tout homme»).

Or ce sont exactement ces passages où la correspondance entre totalitas et l'original arabe est parfaite qui sont les plus intéressants du point de vue métaphysique et théologique. Dans le premier, le terme est utilisé afin de montrer que la forme - qui correspond à la fois à l'espèce, au genre et à la différence qui détermine l'espèce, voire à l'idée de la chose - peut se dire aussi du tout ensemble (li-ğamī ' $\underline{\text { da }}$ lika) : la forme comprend en soi le tout de la chose (ou renvoie à l'universel de la chose) et donc virtuellement toutes ses "parties ${ }^{15}$. Dans le deuxième - sur lequel on s'arrêtera assez longuement - Avicenne présente avec le terme totalitas (al-kulliyya) son idée de création absolue ou instauration $(a l-i b d \bar{a})^{16}$.

L'idée de totalité sert ici à définir - plus ou moins implicitement selon les cas - l'idée d'origine et donc de création absolue. À son tour, cette idée ne peut être intelligible sans renvoyer à deux autres notions: la notion (négative et aporétique) d'inexistence (nihil ou privatio) et le concept d'absolu (en arabe muțlaq: ce qui est absolu et donc aussi «indéterminé»,

14. Avicenna Latinus, Liber de philosophia prima. Lexiques.

15. Le passage propose en réalité un problème de lecture. Le texte arabe se réfère à l'universel [kulliyyat al-kulli] alors que le latin au «tout»; Bertolacci traduit: "Si dice "forma" la specie di qualcosa, il suo genere, la sua differenza specifica ed il loro insieme. L'universalità dell'universale è forma anche delle parti», in Avicenna, Libro della guarigione. Le cose divine, A. Bertolacci (trad.), Turin, Utet, 2007, p. 545; mais cf. Liber de philosophia prima, VI, 4, p. 324, 22-24: «Et dicitur forma species rei et genus eius et differentia eius et hoc totum, quia totalitas totius [kulliyyat al-kull?] forma est etiam partibus»; cf. Ilāh., VI, 4, p. 282, 11-13; dans ma traduction, je lisais kulliyyat al-kull: Avicenna, Metafisica. La scienza delle cose divine dal Libro della guarigione (Kitāb al-Šifä'), O. Lizzini (trad.), Milan, Bompiani, 2006, p. 627: "si dice "forma" [...] della specie della cosa, del suo genere e della sua differenza specifica e di tutto questo insieme; e anche la totalità del tutto è una forma per le parti». Comment comprendre la relation entre l'universel, les espèces et les différences spécifiques dans les termes de la totalité? Avicenne discute de la différence entre le tout (al-kull) et l'universel (al-kullī) en Iläh. V, 2, p. 212, 3-16.

16. Liber de philosophia prima, VIII, 4, p. 396, 24-41. 
«libre», «sans limites»). La première notion exprime le néant - en tant que privation ou non-existence -, voire le manque absolu qui justifierait l'acte créatif de Dieu en tant que tel: si la creatio est absolue, c'est qu'elle est creatio ex nihilo ${ }^{17}$. La deuxième connote un acte - celui qui explique l'origine de l'être - qui, en tant que libre et non limité par d'autres, est absolument premier et instaurateur. L'idée de totalité trouve donc son opposé et presque son synonyme dans les deux pôles du néant et de l'absolu. C'est à la lumière de cette prémisse, tirée de ce dernier texte, que j'essaierai de clarifier le sens de totalitas dans la métaphysique d'Avicenne.

\section{Création absolue}

$\mathrm{Si}$, comme on vient de le voir, le concept de totalité (totalitas / kulliyya) se révèle capital pour l'idée de création, c'est que - inséré dans la tradition aristotélicienne - Avicenne "corrige» Aristote à propos de la question (essentielle pour la métaphysique) de l'origine du monde. C'est un motif connu. Tout en suivant l'idée fondamentale de l'éternité du monde - le tout de l'univers est éternel et n'a donc pas d'origine dans le temps -, Avicenne abandonne Aristote au moment où il pose la question de l'origine du tout, et par conséquent celle du passage du non-être à l'être. En d'autres termes, le monde n'a aucune origine dans le temps, mais cela ne signifie pas qu'il n'y ait pas d'origine tout court. Plus précisément, c'est parce que le monde n'a pas d'origine dans le temps que son origine - sa création peut être définie comme absolue. C'est en ce premier sens en effet, et non pas seulement parce qu'elle relève de la physique, qu'Avicenne rejette la démonstration d'un premier Principe qui serait le moteur du monde (parce qu'il en serait la cause finale), et insiste par contre sur l'idée de la causalité efficiente qui doit être attribuée au Premier Principe précisément en tant qu'il est cause de l'être ${ }^{18}$.

17. Même dans le cas du néant on retrouve dans l'arabe, comme en traduction, des termes différents: 'adam, qui vaut «non-existence» et «privation», la locution lā 'an šay' ou 'an lā šay' (et aussi lā min šay') - non a partir d'une chose - et l'abstrait lays (ou laysa) «nonêtre", à partir duquel la philosophie - par ex. avec les textes de la ps. -Théologie d'Aristote et d'al-Kindī - dérive un positif «être» et «faire être» (aysa, ta'yīs), dont la lexicographie arabe a oublié en général l'existence. J'ai analysé ces thèmes et leur terminologie dans Fluxus..., p. 235-278.

18. Concernant le rapport d'Avicenne à la tradition aristotélicienne, voir D. Gutas, Avicenna and the Aristotelian Tradition. Introduction to Reading Avicenna's Philosophical Works, Leyde, Brill, 2014. Pour la théorie de la causalité à laquelle Avicenne se réfère, C. d'Ancona, "La notion de cause dans les textes néoplatoniciens arabes», Cahiers de la revue de théologie et de philosophie, nº 20, 1999, p. 47-68; C. d'Ancona, "Avicenna and the Liber de Causis: A Contribution to the Dossier", Revista Española de Filosofía Medieval, n ${ }^{\circ}$ 7, 2000, p. 95-114; R. Taylor, «Primary Causality and ibdā' (creare) in the Liber de Causis», in Wahrheit und 
La question sur l'origine, voire sur la transition du non-être à l'être, est, comme on le sait, partie intégrante du milieu philosophique dans lequel Avicenne est immergé. Les textes du néoplatonisme gréco-arabe avaient déjà inséré l'intelligence et son acte dans un dessein métaphysique, dont la question de fond était celle de l'origine du Tout. Et encore, les penseurs qui précèdent Avicenne et avec lesquels il doit se confronter (principalement les chrétiens de l'école d'al-Fārābī, al-Fārābī lui-même et, de façon critique, les théologiens du Kalām et al-Kindī) posaient tous au centre de leurs réflexions la question - qui est aussi religieuse - de la création et de l'origine ${ }^{19}$.

Perçue dans sa valeur absolue, la question de l'être était donc déjà, avant Avicenne, en relation avec les questions du tout et du néant, et c'est dans ce contexte qu'Avicenne recherche une formulation de l'acte divin absolu qui soit satisfaisante sur le plan philosophique. Aussi, comme l'élaboration d'une telle formulation n'est pas, pour ainsi dire, indolore, Avicenne est conduit à questionner d'une part la notion aristotélicienne de cause agente (et in fine les notions d'acte et de puissance sur lesquelles celle-ci est bâtie) ${ }^{20}$, d'autre part l'idée de création élaborée par les théologiens. L'action au sens aristotélicien du terme, tout comme la création telle qu'elle est conçue par les auteurs du Kalām, sont en effet inutilisables si l'on veut rendre compte du caractère absolu de l'acte divin: chacune à sa façon, elles présupposent implicitement un substrat pour l'acte divin et ne peuvent donc pas servir à exprimer véritablement la création ex nihilo, voire une origine qui soit telle par rapport à la totalité des choses. Si l'objet de la création de Dieu est tout - en termes théologiques on pourrait dire qu'il s'agit de chaque chose sauf Dieu lui-même - rien en dehors de l'être de Dieu ne peut être convoqué pour expliquer l'acte divin. Or l'idée de l'action, qui se réalise maintenant sans être auparavant, pose de fait un substrat: dans les termes aristotéliciens, l'action est en fait expliquée grâce au passage de la puissance à l'acte, et ce passage, qui se produit dans le temps, présuppose une matière. Avicenne analyse cette idée en Ilāhiyyāt IV, 2 pour établir que la possibilité de la chose, conçue dans le temps et soumise à l'action, n'est rien d'autre que la matière (le temps implique la matière, et l'agir dans le temps implique

Geschichte. Die gebrochene Tradition metaphysischen Denkens, A. Mensching-Estakhr et M. Städtler (dir.), Würzburg, Königshausen \& Neumann, 2012, p. 115-136.

19. D. Twetten, "Arabic Cosmology and the Physics of Cosmic Motion", in The Routledge Companion to Islamic Philosophy, R. Taylor (dir.), Londres - New York, Routledge, 2016, p. 156-167; M. Chase, "Discussions on the Eternity of the World in Late Antiquity", SXOLH. A Journal of the Centre for Ancient Philosophy and the Classical Tradition, $\mathrm{n}^{\circ} 5$, 2011, p. 111-173, et "Creation in Islam from the Qur'ān to al-Fārābì », in The Routledge..., p. 248-26o.

20. Ilāh., VI, 1, p. 257, 10-14. 
un substrat ${ }^{21}$. La définition de la création dans les termes de l'action est ainsi refusée (plus que dans les termes de l'action, la création devrait être pensée en tant qu'activité $)^{22}$.

Mais le refus de la conception aristotélicienne de l'action s'entrelace avec la polémique théologique. En effet, la théologie présuppose elle aussi implicitement un substrat. Quelles que soient les distinctions des écoles (on ne rentrera pas ici dans les détails qui nous amèneraient à parler de la conception de la «chose» et du néant en tant que «non-chose») ${ }^{23}$, les théologiens conviennent que la création du monde est dans le temps parce que le monde «n'a pas toujours été». L'idée du monde créé est exprimée par un terme qui a une connotation temporelle évidente: muḥdat ou «ce qui est - ou vient à l'être - après n'avoir pas été». L'idée centrale est en fait en

21. Ilāh., IV, 2, p. 181, 7-12.

22. Par ex. Ilāh., IV, 2, 172, 13-173, 12; pour Aristote, Métaphysique, Delta 7, 1017b1-10 et la discussion de Theta. Voir au moins J. Beere, Doing and Being. An Interpretation of Aristotle's Metaphysics Theta, Oxford, Oxford University Press, 2009; Dunamis. Autour de la puissance chez Aristote, M. Crubellier, A. Jaulin, D. Lefebvre et P.-M. Morel (dir.), Leuven, Peeters, 2008, p. 327-351. À l'arrière-fond se trouve la question de l'interprétation et de la transformation du sens de la formule aristotélicienne de l'acte et de Dieu en tant qu'acte pur; K. Trego souligne l'analogie entre puissance et possibilité chez Avicenne dans La Liberté en actes. Éthique et métaphysique, d'Alexandre d'Aphrodise à Jean Duns Scot, Paris, Vrin, 2015. La doctrine sur la causalité agente ou efficiente chez Avicenne, qui interprète la notion aristotélicienne, exigerait une longue analyse; une tentative est offerte par Kara Richardson («Avicenna's Conception of the Efficient Cause», British Journal for the History of Philosophy, $\mathrm{n}^{\circ}$ 21, 2013, p. 220-239), qui a le mérite de rechercher une notion générale chez Avicenne qui puisse comprendre à la fois les causes naturelles et les causes divines. Néanmoins, tout examen exhaustif devrait prendre en considération la distinction qu'Avicenne pose entre puissance et possibilité, d'un côté, et entre être en acte et être actif de l'autre (Dieu est proprement actif et donc réellement agent). De fait, Avicenne déclare que ce qui est dit être agent dans la mesure où il agit et n'agit pas (ou dans la mesure où il peut agir et ne pas agir) n'est pas réellement agent (v. Ilāh., VI, 1, 263, 3-13). Ce qui est réellement ou vraiment agent est ce qui agit toujours et a toujours le pouvoir d'agir. Sur la causalité efficiente chez Avicenne, voir aussi: E. Gilson, «Notes pour l'histoire de la cause efficiente", Archives d'histoire doctrinale et littéraire du Moyen Âge, $\mathrm{n}^{\circ}$ 37, 1962, p. 7-31; M. Marmura, «The Metaphysics of Efficient Causality in Avicenna (Ibn Sina) », in Islamic Theology and Philosophy, M. Marmura (dir.), Albany, State University of New York Press, 1984, p. 172-187; J. Jolivet, «La répartition des causes chez Aristote et Avicenne: le sens d'un déplacement", in Lectionum varietates. Hommage à Paul Vignaux (1904-1987), J. Jolivet, Z. Kaluza et A. de Libera (dir.), Paris, Vrin, 1991, p. 49-65; R. Wisnovksy, «Final and Efficient Causality in Avicenna's Cosmology and Theology", Quaestio, n 2, 2002, p. 97-123; id., «Towards a History of Avicenna's Distinction between Immanent and Transcendent Causes», in Before and After Avicenna, Leyde, Brill, 2003, p. 49-68; T. Kukkonen, «Creation and Causation", in The Cambridge History of Medieval Philosophy, Cambridge, Cambridge University Press, 2010, p. 232-246.

23. M. Chase, «Creation in Islam... »; O. Lizzini, «Il nulla (al-'adam), l’inesistente (al-ma'dùm), la cosa (al-shay'): note intorno alla terminologia e alla dottrina della creazione dal nulla nel pensiero islamico ", in Discussioni sul nulla tra Medioevo ed Età moderna, M. Lenzi et A. Maierù (dir.), Rome, Lessico Intellettuale Europeo, 2009, p. 63-103. 
théologie celle d'un monde qui est "après n'avoir pas été», et qui est donc forcément dans le temps (qui n'existe plus - est passé - avec la venue à l'être du monde). Plus que d'une création ex nihilo, le concept théologique de la création se révèle ainsi - comme Avicenne le souligne - une création post nihil qui pourrait peut-être expliquer un passage temporel, voire une transformation, mais qui ne pourrait en aucun cas rendre compte de l'idée d'une origine "absolue» du tout. Pour précéder le monde, le nihil devrait soit exister (il s'agirait alors de la matière, comme dans l'action, ou de la possibilité, comme dans les théories mu'tazilites de la création), et on aurait donc un substrat, soit ne pas exister, et donc évidemment ne pas être précédent ${ }^{24}$.

L'idée philosophique d'action, comme l'idée théologique de création dans le temps, sont donc philosophiquement non satisfaisantes. Les critiques adressées à ces deux conceptions vont souvent se confondre, même si la polémique contre la théologie apparait plus explicitement que celle consacrée à la conception aristotélicienne (la définition aristotélicienne de l'agir est d'ailleurs parfaitement acceptée en ce qui concerne le monde sublunaire). Un texte du jeune Avicenne - le Livre de la genèse et du retour - offre une bonne perspective sur la question : l'idée de la création y est explicitement liée à celle du caractère absolu qui doit être attribué à la causalité divine ${ }^{25}$, et la création y est définie - selon le sens que «les sages» attribuent au terme en tant que "faire être en permanence quelque chose qui, en soi, n'est pas" (idāmatu ta'yīs mā huwa bi-d̄âti-hi laysa) ${ }^{26}$. Ce «faire être en permanence», qui coïncide évidemment avec l'émanation, ne dépend d'aucune autre cause que le Premier lui-même (là tata'allaqu bi-'illatin gayri dâti l-awwali) ${ }^{27}$ et conduit donc à l'exclusion de toute médiation, que ce soit celle d'une matière (mādda), d'un outil (āla), d'une chose quelconque (ma'nā), ou, selon une autre interprétation possible, d'une intention $\left(m a^{\prime} n \bar{a}\right)$. Cet "acte», cet «agir» $\left(f^{i} l\right)$, affirme Avicenne, est meilleur ou plus noble (afḍal) que ce que l'on entend habituellement par «action» $\left(f^{\imath} l\right)$. Pour cette raison,

24. Pour la théologie, tout dans le monde et le monde lui-même sont muḥdat, c'est-à-dire un être adventice «qui vient à l'être» et qui, venant à l'être, est aussi destiné à cesser d'être, là où Dieu (et Dieu seulement) est qadìm «éternel» (ou «pré-éternel»), Il ne participe pas du temps. Voir les références dans D. Gimaret, Les Noms divins en Islam. Exégèse lexicographique et théologique, Paris, Cerf, 1988. Pour les correspondances dans le langage philosophique platonicien et néoplatonicien voir M. Chase, "Creation in Islam...».

25. K. al-Mabda' wa-al-Ma'ād, A. Nūrānī [éd.], Téhéran, Mu'assasa Matal'at Daneshga Mc Gīl, 1342h/1984, 76, 8-14; Livre de la Genèse et du Retour, Y. Michot (trad.), Oxford, Oxford Center for Islamic Studies, 2002, p. 55.

26. Mabda', p. 77, 10 (avec laysun); Genèse, p. 55. Pour ta'yīs v. Ilāh., VIII, 3, p. 342, 17; pour toute la discussion autour de ibdā' chez Avicenne, Lizzini, Fluxus..., p. 257-278.

27. Littéralement on aurait: «l'essence du Premier »; Mabda’, p. 77, 11; Genèse, p. 55. 
on doit lui donner un nom approprié, et ce n'est que temporairement, ou métaphoriquement, qu'on peut l'appeler «instauration» ou «création»:

Quant aux Sages, ils veulent dire par " $i b d \bar{a}^{\prime} »$ le faire-être en permanence de quelque chose qui, par son essence, n'est pas, un faire-être en permanence ne se rattachant à nulle autre cause que l'essence du Premier, ni à une matière, ni un instrument, ni une intention, ni une médiation ${ }^{28}$.

Si Avicenne critique le concept de création dans le temps, utilisé par les théologiens, c'est exactement parce qu'il en tire les conséquences extrêmes. Si les théologiens identifient la création en tant que telle avec un acte produit par la cause en absence de toute médiation matérielle, les philosophes excluent toute médiation (celle d'une matière, mais aussi celle d'un instrument et d'une intention), pour éliminer également le temps et tout substrat qui, dans le temps, existerait ${ }^{29}$.

On l'a vu: l'exigence sous-jacente majeure au discours d'Avicenne est celle d'exprimer l'origine absolue, voire totale, de l'être. C'est en vertu de cette exigence que l'on ne peut pas concevoir la création dans le temps. Aux arguments déjà repris (le néant ne peut pas être pensé, et son antériorité non chronologique ne peut donc pas être incluse dans la dimension de la création; l'inclure signifierait d'ailleurs forcément penser l'action divine en relation à une potentialité et donc à une matière), on doit ainsi ajouter un argument qui concerne le Principe lui-même: dire que Dieu crée «à partir du néant » impliquerait l'affirmation d'un «moment de la création » et d'un «moment de la non-création». On déterminerait de la sorte un domaine d'application pour l'action divine qui se trouverait ainsi niée dans son caractère d'absolue ${ }^{30}$. Au contraire, précisément parce qu'elle n'assume aucun «moment», la conception philosophique de l'instauration, qui coïncide avec l'émanation, le flux, rend compte d'un enlèvement, pour ainsi dire, absolu de la non-existence, qui ne gagne ainsi aucun poids ontologique, ne pose aucun substrat, et ne conduit donc pas à la contradiction.

La conception philosophique supprime le non-être de façon cohérente parce qu'elle ne le pose pas et laisse entrevoir l'idée de l'émanation, voire de la dépendance logico-ontologique du monde par rapport au Principe, comme la seule interprétation de l'acte instaurateur divin qui soit correcte du point de vue de la rationalité. C'est en fait par l'idée de continuité qu'Avicenne fait passer le concept de l'absolu de l'agir divin face à la totalité, en récupérant

28. Mabda', p. 77, 10-11; cf. Genèse, p. 55 (traduction par Y. Michot, légèrement modifiée).

29. Mabda', p. 76, 15-77, 2.

30. Ibid. 
ainsi ce que la nature conditionnelle du possible (le Premier agit sur le possible, qui est en ce sens "non-être») semblait en quelque sorte nier ${ }^{31}$.

Ce discours, dont on n'a suivi ici que les prémisses, est comme inclus dans le passage des Ilāhiyyāt que l'on a déjà mentionné, où la notion de totalitas (al-kulliyya) apparaît avec un sens authentiquement métaphysique. Ce passage, essentiel à la question de «l'enlèvement» - pour ainsi dire - absolu du néant, fait partie de la discussion de la causalité qui est au cœur du Traité VIII de la Métaphysique. Avicenne relie la discussion à l'examen de la relation de causalité, à savoir la «relation qui donne raison de l'être causé» (nisba ma'lūliyya) et qui ancre les effets dans les causes, en les raccordant enfin à la première cause absolue. Il s'agit de la discussion au cours de laquelle Avicenne établit la finitude des causes et - ce qui est plus intéressant ici - en déduit la causalité qui doit être attribuée au Premier, laquelle doit rendre compte d'un faire être absolu, et non pas d'un certain faire être. L'existence du Principe nécessaire, déjà établie à partir de la nécessité d'une cause finale de la série ${ }^{32}$, permet de déduire, à partir des propriétés qui doivent être attribuées au Principe, celles du possible. Tout ce qui est autre par rapport au Principe en est différent, est donc possible dans l'essence, et causé, parce que cela « reçoit» (qābil) l'existence (ou l'obtient: est acquirens esse) ${ }^{33}$. Il devient donc possible de définir la création dans les termes de la totalité.

Voyons le premier élément:

$[M]$ anifestum est quod necesse esse unum numero est, et patuit quod, quicquid aliud est ab illo, cum consideratur per se, est possibile in suo esse, et ideo est causatum et paene innotuit quod in causalitate sine dubio pervenitur ad ipsum. Unde quicquid est, excepto uno quod est sibi ipsi unum et ente quod est sibi ipsi ens, est acquirens esse ab alio a se, per quod est sibi esse, non per se $^{34}$.

Sont ici présentées quelques-unes des thèses fondamentales de l'ensemble du système avicennien. On reconnaît premièrement l'idée que la causalité

31. O. L. Lizzini, Fluxus..., p. 89-147 et p. 157-162.

32. Dans les passages précédents (Ilāh., VIII, 3, p. 340, 14-342, 5), Avicenne a démontré la finitude des causes finales: si la cause finale ou perfective (al-tamāmiyya) est celle en vue de laquelle toutes les autres choses existent, il est clair que la cause finale authentique ou première ne peut pas faire elle-même l'objet d'une autre cause; dans ce cas, elle serait «en vue d'autre» et ne correspondrait pas à la définition qu'on en a donnée. Le caractère fini de la série causale apparaît donc comme déductible à partir de la position même de la cause finale: là où on a posé la cause finale, le recours à l'infini se montre impossible.

33. Liber de philosophia prima, VIII, 4, p. 395, 18-396, 23. Selon la lecture actuelle du texte: "obtient ( $\left.n \bar{a}^{`} i l\right)$ l'existence»; mais voir la correction de Bertolacci dans Libro della guarigione. Le cose divine..., p. 124.

34. Liber de philosophia prima, VIII, 3, p. 395, 18-396, 23 ; Ilāh., VIII, p. 342, 6-14. 
du Premier consiste à donner l'existence et qu'en cela réside son caractère de créateur ou «instaurateur» (mubdi $)$ du monde. Ce don de l'existence comporte deuxièmement la composition du causé, en essence et existence. L'analyse du causé (qui se fait en réalité toujours a posteriori) révèle un causé qui en soi est possible et donc n'est pas (laysa) et est (aysa) (et donc est nécessaire) en vertu du Principe, selon la correspondance fondamentale - et pourtant problématique - entre la possibilité et l'inexistence qui régit l'ontologie avicennienne: la possibilité qui appartient en soi au causé est identique à la non-existence que la chose en soi mérite (ou à laquelle elle a droit). L'identification entre non-existence et possibilité, sur laquelle Avicenne fonde son idée du flux, permet d'affirmer que l'antériorité de l'inexistence par rapport à l'acte instaurateur divin est essentielle et non temporelle ${ }^{35}$. On retrouve ici le thème de la création:

Et haec est intentio de hoc quod res est creata, scilicet quod est recipiens esse ab alio a se et habet privationem quae certificatur ei in sua essentia absolute, non quod certificetur ei privatio propter suam formam absque sua materia, vel propter suam materiam absque sua forma, sed per suam totalitatem. Igitur si sua totalitas non fuerit simul cum debito essendi datorem esse, tunc, si posueris ipsum remotum ab ea, debebit esse privatio eius cum sua totalitate; quod est oppositum ad ipsam esse a datore essendi ipsam cum sua totalitate. Igitur non erit aliqua pars eius cuius esse praecedat alteram secundum considerationem huius sententiae, sed quia nec materia eius nec forma eius, si fuerit habens materiam et formam, totum igitur respectu primae causae creatum est ${ }^{36}$.

Il n'y pas de néant avant la création. On l'a déjà remarqué: admettre un temps où il n'y a rien signifie accueillir de façon contradictoire l'idée d'un temps où «il y a le rien». Tout cela est clarifié dans la suite du passage: une non-existence qui précède dans le temps la création suppose contradictoirement que le néant ait une existence, et donne ainsi un "pouvoir» à l'inexistence; ainsi la création (dare esse, creatio, inceptio) n'aurait qu'un sens relatif, et ne serait donc plus une création ${ }^{37}$ :

Sed dare esse ei quod est ab ipso non est tale dare esse quod omnino prohibeat privationem a substantiis rerum, sed est tale esse quod prohibet privationem absolute [et est] in eo quod potest sustinere aeternitatem, et haec est creatio absoluta et esse absolutum, non esse aliquid. Omne enim quod coepit ab illo uno est, et illud unum est dans ei inceptionem, eo quod id quod incipit est id

35. Ilāh., IV, 1, p. 164, 18-165, 9.

36. Liber de philosophia prima, VIII, 4, p. 396, 24-35. Ilāh., VIII, 3, p. 342, 10-15.

37. Il ne s'agit pas d'un "certain faire être», mais de "faire être au sens absolu»; Ilāh., VIII, 3, p. 342,17 . 
quod est post non esse. Sed si istud post fuerit temporale, tunc praecedet illud ante, sed remevetur hoc ante cum illud incipit esse; erit igitur res appropriata sic quod eam praecessit ante, et non est nunc; igitur non proveniet esse aliquid, nisi ante illud fuerit aliud quod destruatur, ipso iam essente. Igitur inceptio de non absolute quae est creatio erit cassa, non habens intellectum. Igitur post quod est hic est post quod est per essentiam, quia id quod est rei ex seipsa prius est eo quod est ei ex alio a se; postquam autem est ei ex alio esse et debitum essendi, tunc habet ex se privationem et possibilitatem, et fuit eius privatio ante esse eius $<$ et esse eius $>$ post privationem eius prioritate et posterioriate per essentiam. Igitur omnis res, excepto primo, est postquam non fuit ens, quantum in se est ${ }^{38}$.

L'idée théologique de création à partir du néant est donc définitivement dissoute. Pourtant, le passage pose implicitement une autre question. Une fois admis que l'idée de la création dans le temps n'est pas une création, on doit en effet comprendre quel rôle il faut attribuer à la création ou production dans le temps. Le passage le dit d'ailleurs explicitement: la création en dehors du temps est une donation de l'être absolue qui explique l'être créé en tant qu'éternel («tale esse [... est] in eo quod potest sustinere aeternitatem») et qui ne pose aucune matière (ou forme) avant la chose créée elle-même ("non quod certificetur ei privatio propter suam formam absque sua materia, vel propter suam materiam absque sua forma»). Avicenne doit établir une distinction entre la création absolue ou «instauration» et la production dans le temps (qui exige une matière). Et ainsi, en expliquant quelle différence maintenir entre «fait advenir» ou "fait venir à l'être» ou «produit dans le temps» (muḥdaț) et «créé» ou «instauré» (mubda), Avicenne obtient un double résultat. Avec sa réfutation du concept théologique de création, Avicenne fonde d'un côté sa propre idée de flux divin (où l'antériorité ontologique du Premier et l'éternité du monde sont impliquées); de l'autre, il rend raison du trait qui, ontologiquement, distingue la première intelligence causée - et par extension le monde supralunaire - et les êtres du monde sublunaire. Si les intelligences sont des êtres «instaurés» (mubda'ät), les êtres composés de matière et de forme sont des êtres "produits» (muḥdațāt) et impliquent une antériorité de la possibilité qui coïncide avec la potentialité de la matière.

Ainsi, si la conception avicennienne doit expliquer l'origine absolue, voire l'origine de la totalité de l'être, c'est néanmoins plutôt une articulation de la totalité qui est ainsi reconnue, où l'opposition Principe-monde (Principe-totalité des choses) se dit dans la distinction des êtres simples - qui sont simplement et éternellement causés dans leur totalité - et les

38. Liber de philosophia prima, VIII, 4, p. 396, 35-397, 52. Ilāh., VIII, 3, p. 342, 15-343, 6. 
êtres qui, produits dans le temps, présupposent un substrat qui soit dans le temps. Si les premiers ne sont doubles (ou composés) que par rapport à la composition ontologique fondamentale de l'essence et de l'existence, les seconds impliquent une composition ultérieure où la matière et la forme viennent à être convoquées; en ce sens, les êtres temporellement déterminés ne sont pas causés dans la totalité de leur être (la matière étant déjà existante afin de recevoir les formes). La notion de néant fonctionne ici comme à l'inverse par rapport à celle de totalité: si on a une conception de la totalité, c'est qu'on pose l'être comme un tout à l'opposition duquel il y a le néant ou - plus précisément - il n'y a rien.

Dans le texte que l'on vient de lire, Avicenne définit, en somme, l'instauration ou création absolue (creatio absoluta; al-ibdā'al-muțlaq) comme l'acte qui crée les choses dans leur totalité (ou la chose dans sa totalité: per suam totalitatem; bi-kulliyyati-hi), mais il distingue également les choses produites dans le temps (par la conjonction de la matière et de la forme) de celles qui sont proprement créées ou instaurées ${ }^{39}$.

\section{Privation, bien absolu et mal : la totalité du bien}

C'est donc à partir des notions (ou de non-notions) de néant et d'absolu qu'Avicenne élabore l'idée de la création ou instauration continue, voire du flux, et donc celle de totalité qui la soutient. L'analyse de la légitimation du bien du monde, qui inclut ou est aussi un mal, offre une autre perspective. Je n'insisterai pas sur les détails des passages fonctionnels de ce discours (il s'agit, pour l'essentiel, de Métaphysique IX, 6) ${ }^{40}$. Je me limiterai à signaler les lieux les plus importants. Le cœur de l'argument d'Avicenne autour du mal est la relativisation qu'il en établit: le mal est nécessairement relatif et il est donc une absence ou privation relative à l'être auquel il appartient, voire dont il est l'inexistence. Il est donc nécessairement lié à la puissance et à la matière ${ }^{41}$.

Avicenne précise cependant que le mal n'est pas une non-existence ou un manque quelconque, mais seulement la non-existence de ces perfections

39. Liber de philosophia prima, VIII, 3, p. 396, 24-397, 52 ; Ilāh., p. 342, 6, p. $343,6$.

40. Il existe une belle étude de Carlos Steel dans laquelle la conception d'Avicenne est comparée à celle de Thomas d'Aquin: "Avicenna and Thomas Aquinas on Evil ", in Avicenna and His Heritage (Actes du colloque de Louvain, 8-11 septembre 1999), J. Janssens et D. De Smet (dir.), Leuven, Leuven University Press, 2002, p. 171-196.

41. Iläh., IX, 6, p. 414, 17-417. Les premiers sens du mal qu'Avicenne isole sont en effet l'absence (en raison du manque de quelque chose) et la souffrance, qui est due à la présence d'une cause (qui peut à la fois être soit distinguée de la chose - comme le nuage est la cause du fait que la maturation du fruit est empêchée - soit conjointe à elle - comme la plaie est jointe à la personne qui en souffre). 
qui doivent ou devraient appartenir à un sujet donné. Le néant absolu et donc le mal absolu n'existent, en effet, que pour le langage. Le mal universel ne peut pas exister, parce que le mal est lié à la puissance et à la matière, et est donc toujours relatif: il est toujours lié à quelque chose d'existant. En effet, si le mal universel existait, il n'y aurait pas de place pour le bien: la totalité serait négative et ne pourrait prévoir rien d'autre que cette négativité; finalement elle ne serait même pas ${ }^{42}$. Le mal peut en effet accompagner la matière de deux façons. En soi d'une part, si la matière est disposée selon une mauvaise complexion. Dès lors, la cause du mal est à rechercher dans le principe récepteur lui-même. En vertu d'autre chose que la matière elle-même d'autre part, si elle empêche la réalisation de la perfection de la chose (comme le nuage empêche la maturité du semis) ou si elle la détruit (comme la grêle détruit la récolte). Ces distinctions permettent à Avicenne d'affirmer la portée relative du problème du mal, là où la cause du mal est tout entière en dessous de la sphère de la Lune. Le mal, en fait, ne concerne jamais l'espèce qui est un tout en soi-même, et est en tant que telle parfaite. Le mal ne touche que les individus, et encore ne touche-t-il qu'une partie des individus, jamais leur majorité ${ }^{43}$.

Or, ce discours sur la relativité du mal intéresse la question de la totalité et de l'absolu, précisément parce qu'il montre que l'objet de la création divine est la totalité des choses et doit donc comprendre aussi celles qui sont liées à l'imperfection et au mal. Le mal (avec sa relativité) est en ce sens inscrit dans le décret divin: en étant un conséquent nécessaire du bien (littéralement «du besoin de bien»), il est "voulu» par accident ${ }^{44}$. L'exemple qu'Avicenne utilise fréquemment à ce propos est celui du feu qui, tout en étant en soi un bien, est aussi ce qui peut brûler - ou ce qui brûle en effet - le manteau d'un homme pieux. Le Principe - et on a ici la légitimité éthique du mal - ne peut pas renoncer à un mal relatif (le feu), parce que cela signifierait non seulement l'absence du bien relatif auquel il est lié, mais aussi l'absence, voire l'inexistence du bien absolu qui en est la cause: de la sorte, pour éviter une inexistence, il y en aurait deux ${ }^{45}$. La procession

42. Ilāh., IX, 6, p. 421, 9-10; Liber de philosophia prima, p. 504, 41-43: «quod autem totum est malum vel in quo praevalet vel est aequale, nondum invenitur".

43. Ilāh., IX, 6, p. 417, 6-18; ce n'est qu'une forme spécifique de mal, d'ailleurs, qui touche les individus: le mal peut concerner ce qui est nécessaire à la perfection de la chose, ce qui est presque nécessaire, mais aussi ce qui est superflu (un exemple de ce genre de mal est l'ignorance d'une discipline scientifique; bien qu'elle soit regrettable en soi, elle n'est mal que dans le cas de cet individu précis qui perçoit la nécessité de connaître cette discipline).

44. Ilāh., IX, 6, p. 418, 1-422.

45. En d'autres termes, si, pour éviter le mal (qui est une non-existence relative), on renonçait au bien relatif dont il est le conséquent, on renoncerait en même temps au bien parfait - et dans un certain sens absolu - du monde céleste, dont le bien relatif ou prédominant 
du flux est justifiée et est donc en soi un «bien», même dans le mal que sa dernière expression implique, ou bien exactement dans le mal (relatif) que sa dernière expression implique: le mal est donc «voulu » et en ce sens choisi et porté à l'acte par le Nécessairement Existant. Le bien "relatif» a finalement une nécessité intrinsèque: le bien du monde sublunaire (avec le mal qui y est relié) doit, en tant que possible, être réalisé, en vertu du "principe de plénitude» qui réglemente l'ensemble du système avicennien. Avicenne énonce en fait clairement que «même si» on ne voulait pas prêter attention au calcul "des deux inexistences", le monde sublunaire (voire le bien et le mal que celui-ci représente) s'avérerait nécessaire ${ }^{46}$. J'ai déjà noté ailleurs que la distinction qu'Avicenne introduit entre un bien absolu et un bien prédominant conduit à affirmer que le véritable sens du bien absolu consiste dans la réalisation de la totalité des possibles. Ce n'est qu'apparemment que le «bien absolu» est un bien auquel aucun mal n'est mélangé; le vrai bien «absolu» ne réside pas dans le monde céleste, mais dans la réalisation du possible en tant que tel et cela comprend à la fois le monde céleste et le monde en dessous de la sphère de la Lune ${ }^{47}$.

En ce sens, l'émanation est ce qui donne une fondation non seulement ontologique, mais aussi éthique, de la totalité de l'être, dont la raison ultime est la totalité elle-même ${ }^{48}$. Une fois que le bien absolu a été réalisé, il reste (yabqā) une possibilité, qui est celle du monde sublunaire. Le vide que la possibilité non réalisée laisserait est inconcevable. Si la possibilité du monde sublunaire n'avait pas été réalisée, l'ordre du bien serait en fait bouleversé. Ainsi, comme Avicenne lui-même le déclare, même sans tenir compte des arguments mentionnés - et donc sans évaluer l'avantage qu'une seule non-existence représente par rapport à deux - la nécessité du monde est incontournable: une fois que le monde au-dessus de la Lune existe, le monde qui est en dessous - et qui n'est que «possible» - doit aussi exister. L'argument (dont l'origine est plotinienne ${ }^{49}$ ) montre donc que le bien absolu

du monde d'ici-bas n'est que le conséquent. Si le Premier principe «choisit» le bien, cela n'advient en fait qu'à partir d'une alternative qui lui est, pour ainsi dire, déjà donnée. Le Principe choisit le mieux parce qu'il opte pour ce qui ressemble à un mal mineur (ou littéralement «meilleur»: hayr al-šarrayn: Ilăh., IX, 6, p. 418, 18) entre deux «maux» qui se présentent comme le résultat à la fois immédiat et inévitable de son "agir » : le moindre mal est la conséquence de l'instauration du monde, tandis que le mal majeur serait la conséquence de la non-action du Principe. La non-existence du bien relatif et du bien absolu (voilà la formule de deux «inexistences» ou deux privations: 'adamān) est un mal pire que la non-existence du bien absolu.

46. Ilāh., IX, 6, p. 418, 12-419, 4.

47. O. L. Lizzini, Fluxus..., p. 300-315.

48. Ibid., p. 278-315 et O. L. Lizzini, «"A Mysterious Order of Possibles"...».

49. Plotin, Enn., IV , 8, 3. C'est le vrai principe de totalité, le principe de plénitude. 
se trouve dans la réalisation de tout ce qui est possible. La totalité de l'être est en soi entièrement un «bien» (même si elle implique un certain mal).

\section{En guise de conclusion}

Ce sont donc les deux notions d'absolu et de non-existence qui se trouvent incluses plus ou moins implicitement dans la notion de totalité. Avicenne présente l'instauration ou création absolue - le terme est $i b d \bar{a}^{c}$ - en utilisant précisément le concept de totalité, la création étant l'acte qui pose la chose dans l'être ou qui fait être la chose dans sa totalité (bi-kulliyyati-hi; per suam totalitatem). Le plan de l'être (et donc de l'instauration) est celui du tout - de la totalité et de l'absolu - qui, en tant que tel, ne peut pas être décomposé en parties et qui ne tolère pas qu'on pose un substrat (comme on devrait le faire si on posait la matière et donc le temps). Et cela même si - il convient de le rappeler - l'être instauré dont on fait ici mention n'est jamais vraiment indépendant de la composition. La composition a pour Avicenne un sens métaphysique fort qui va au-delà de la connexion matière-forme rappelée dans ce passage: tout ce qui est produit est composé et est, donc, un «double» ${ }^{50}$.

Cela nous ramène à la distinction fondamentale de l'ontologie d'Avicenne - celle de l'essence et de l'existence - une distinction qui, pour être vraiment comprise, devrait être toujours et nécessairement conçue rétroactivement, $a$ posteriori, à partir de la chose qui est pour ainsi dire déjà existante. La composition est, en fait, toujours composition de possibilité et d'existence ou de nécessité. Elle l'est au-delà ou en dehors du temps (et donc forcément a posteriori), lorsque les choses qui sont considérées sont celles du monde céleste - le monde intellectuel - ou lorsque les choses sublunaires sont conçues d'un point de vue métaphysique. Elle l'est dans le temps, lorsque les choses sont considérées en tant que telles dans le monde sublunaire: la composition concerne alors la matière et la forme, la possibilité dans le temps étant la matière ${ }^{51}$. Sur le plan métaphysique, la contrepartie de la notion d'émanation est un absolu indéterminé, car ce qui doit être expliqué est le tout. Par contre sur le plan physique, lorsqu'il s'agit d'expliquer non pas la transition générique et aporétique qui va du non-être à l'être, mais la transition du «non-être quelque chose » à l'être « quelque chose de déterminé», la notion d'absolu indéterminé ou de possibilité est insuffisante. Elle est donc remplacée par l'idée d'une puissance articulée, diversement modulée, et en

50. Ilāh., I, 7, p. 47, 18-19: «Seul le [nécessairement existant] est singulier; ce qui n'est pas lui est double et composé» («composé» pourrait être une glose).

51. Ilāh., IV, 2. 
quelque sorte déjà déterminée: la matière et sa préparation ${ }^{52}$. Il faut donc insister sur ce point: la totalité du monde est composition. Elle est composition d'essence et d'existence, et dans le monde sublunaire elle devient aussi composition de matière et de forme. Par conséquent, même le néant et la non-existence sont comme doubles. Ils se rapportent à la matière quand il s'agit de ce qui vient à l'être dans le temps ${ }^{53}$. Ils sont relatifs à l'essence même de la chose, là où selon Avicenne la cause n'a pas de pouvoir sur l'inexistence qui appartient en soi à la chose (voire sur la non-existence de la chose qui équivaut à son essence même ${ }^{54}$. Mais l'examen du concept de totalité chez Avicenne, fût-il exprimé de façon implicite ou explicite, permet aussi de réaffirmer la conception du Premier: le Principe est, quant à lui, incomposé et un, car Il est seulement être ou existence sans limitation et donc sans la limitation «essentielle» qui dépendrait de l'essence ou quiddité55. Si, pour Plotin l'Un est au-dessus de l'être, pour Avicenne le Principe un est au-dessus ou au-delà de la quiddité; toute la conception avicennienne de l'être qui n'est pas et ne concerne pas le Principe repose chez Avicenne sur la scission - et donc sur la composition - des deux éléments conceptuels qui composent l'être des choses: l'essence et l'existence, fusionnées dans une totalité qui s'oppose à la simplicité du Premier principe ${ }^{56}$.

52. O. L. Lizzini, Fluxus..., chap. IV.

53. L'inexistant est tel seulement quand il n'est pas encore causé, et coïncide finalement avec le possible lui-même (un possible qui devient cependant «impossible» en l'absence d'une cause). Pour les détails et les textes, voir de nouveau O. L. Lizzini, Fluxus..., p. 163-177.

54. Voir le passage cité supra : «C’est ce qui veut dire que la chose est créée, i.e. recevant l'existence d'un autre; elle a un non-être qu'elle mérite en elle-même absolument; non pas qu'elle mérite le non-être par sa forme sans sa matière, ou par sa matière sans sa forme, mais par sa totalité. Si sa totalité n'est pas accompagnée par le faire exister de ce qui la fait exister et que l'on suppose qu'elle est séparée de lui, alors son non-être est nécessaire dans sa totalité. Par conséquent son existentialisation provient de ce qui la fait exister dans sa totalité. Dès lors il n'y a pas une partie en elle qui précède son existence par rapport à cette intention, ni sa matière ni sa forme, si elle possède une matière et une forme. Par conséquent, le tout par rapport à la Cause première est créé $(m u b d a)$ et l'acte avec lequel ce qui existe à partir de la [Cause première] est fait exister, n'est pas tel qu'il rende absolument le non-être capable d'avoir un pouvoir sur les substances des choses. C'est un [acte] de faire exister qui, dans ce dont on dit l'éternité, rend impossible l'inexistence en absolu : c'est l'instauration $(i b d \bar{a})$ [ou création absolue] et le faire être ( $\left.t a^{\prime} y \bar{y} s\right)$ absolu et non pas un certain faire être», Ilāh., VIII, 3, p. 342, 10-17. Possible et inexistant coïncident. Libro della guarigione. Le cose divine..., p. 640 et les corrections proposées p. 124; Metafisica..., p. 778-779.

55. Pour la prémisse logique de la distinction entre séparation et négation qui soutient l'affirmation de l'existence une du Premier chez Avicenne, P. Porro, «Immateriality and Separation in Avicenna and Thomas Aquinas", in The Arabic, Hebrew and Latin Reception of Avicenna's "Metaphysics», A. Bertolacci et D. Hasse (dir.), Berlin, De Gruyter, 2012, p. 275-307.

56. Pour Avicenne le Principe est un, car il est au-dessus ou au-delà de la quiddité, mais non pas au-dessus de l'être ou de l'existence (et c'est d'ailleurs ici que s'ouvre la question de l'analogie de l'être). Avicenne s'écarte en ce sens de Plotin, même s'il interprète en ce sens 
Un passage où l'on doit reconnaître - peut-être - une (non-)occurrence du terme totalitas doit être mentionné à ce propos (même si, bien sûr, toute affirmation devra être confirmée à la lumière de l'édition critique du texte de la Métaphysique d'Avicenne ${ }^{57}$ ). Dans le premier traité du Liber de philosophia prima $(\mathrm{I}, 7)$, tout au long d'un discours qui concerne le caractère essentiel de la nécessité en tant qu'attribut du Premier Principe, on lit que: "necesse esse unum est in nomine $"{ }^{58}$. La locution in nomine semblerait traduire l'arabe bi-l-kalima, la graphie de bi-l-kalima - qui se retrouve dans quelques manuscrits - étant bien proche de celle de bi-l-kulliyya «in totalitate», qui est ce qu'on lit dans l'édition actuelle du texte (l'édition du Caire) ${ }^{59}$. Si l'édition confirme la lecture du texte latin, selon l'hypothèse de Bertolacci ${ }^{60}$, on aura ici la proclamation explicite de l'unité absolue du Principe, excédant la totalité du monde qui est toujours composition. $\mathrm{Si}$, par contre, c'est bien en rapport à la totalité qu'il faut comprendre l'unité du Principe, il faudra se demander si cette totalité a un sens purement adverbial, inoffensif et neutre du point de vue métaphysique (le Principe étant entièrement un) ou bien si on peut y voir une référence propre (et aporétique) à la non-composition du Premier et à la composition de la totalité métaphysique.

Il ne s'agit pas d'affirmer quelque chose de nouveau, mais d'insister peut-être sur la formulation correcte pour exprimer la théorie ontologique avicennienne: d'une part, que tout ce qui n'est pas le Principe doit être en soi un double et donc un composé (qui n'est pourtant pas pour cela un «tout» au sens technique où l'on peut, voire l'on doit distinguer des "parties»" ${ }^{61}$ ); d'autre part, et pour ainsi dire à l'inverse, que c'est ici précisément qu'il faut inscrire la définition du Premier principe: le Principe est en effet incomposé

l'hénologie plotinienne. D. De Haan, «The Doctrine of the Analogy of Being in Avicenna's Metaphysics of the Healing», The Review of Metaphysics, n 69, 2015, p. 261-286.

57. Voir le projet de A. Bertolacci, Philosophy on the Border of Civilizations and Intellectual Endeavours.Towards a Critical Edition of the Metaphysics (Ilāhiyyāt of Kitāb al-Šifä') of Avicenna (Ibn Sin̄a), Pise, Scuola Normale Superiore, < http://www.avicennaproject.eu/ index.php?id=1 $>$.

58. Ilāh., I, 7, p. 47, 6: «Par conséquent le nécessairement existant est un entièrement (bi-lkullliyya) non comme les espèces sous un genre». Selon la suggestion de Bertolacci: «le nécessairement existant est un nominalement (bi-l-kalima / in nomine)».

59. La Métaphysique du Shifä', t. I [Livres I à V ] et t. II [Livres de VI à X], 2e éd., G. Anawati (trad.), Paris, Vrin, 1978 et 1985. Anawati traduisait: «par conséquent le nécessairement existant est un, entièrement", ibid., t. I, p. 121.

60. Bertolacci traduit en effet que le Principe nécessaire est «uno nel vero senso della parola». Cf. Libro della guarigione. Le cose divine..., p. 211.

61. A. Bertolacci, «The Distinction of Essence and Existence in Avicenna's Metaphysics: The Text and Its Context», in Islamic Philosophy, Science, Culture, and Religion. Studies in Honor of Dimitri Gutas, F. Opwis et D. C. Reisman (éd.), Leyde, Brill, 2012, p. 257-288. 
et un, car il n'est rien d'autre qu'être ou existence, sans limitation ou sans partition, et donc - on l'a vu - sans aucune limitation dépendant de l'essence.

On retrouve donc les questions de l'univocité et de la hiérarchie (et finalement de l'analogie de l'être) que l'on a évoquées tout au début. Sans pouvoir ici en discuter les enjeux, on doit remarquer qu'elles se relient exactement au thème de la totalité. La troisième section du quatrième livre de la Métaphysique est en ce sens un texte à la fois explicite et peu souvent mentionné. En reprenant les suggestions du néoplatonisme, Avicenne y distingue trois genres d'êtres hiérarchiquement ordonnés: l'être de ce qui est «imparfait», l'être de ce qui est «suffisant» et l'être de ce qui est tout ou «complet». Ce qui est imparfait est au niveau le plus inférieur de l'existence; c'est ce qui existe en tant qu'insuffisant: son être (possible) n'est pas complètement réalisé; littéralement Avicenne parle de ce qui est nāqiṣ: «déficient", "défectueux» et donc «imparfait», et il se réfère évidemment aux entités imparfaites du monde sublunaire, chacune desquelles a besoin - voilà le point sur lequel la définition insiste - de ce qui est autre que soi-même pour réaliser ses propres perfections l'une après l'autre ${ }^{62}$; c'est le monde de la nature et de la composition hylémorphique. Au niveau supérieur, Avicenne place ce qui est suffisant (al-mutakăfî̀) et ensuite ce qui est complet ou parfait (tāmm) dans son existence. La réalisation de l'être (et en ce sens ses parties) est alors soit donnée de façon suffisante, soit donnée de façon complète. À ces ensembles qui réalisent le tout de leur être, quoique de façon différente, correspondent les entités célestes (respectivement les Âmes et les Intelligences), voire les entités instaurées (al-mubda'āt) qui se comprennent à partir de la composition ontologique fondamentale de l'essence et de l'existence. Finalement, Avicenne pose au sommet de cette hiérarchie ce qui est "plus que complet» ou littéralement "au-dessus de la complétude" (fawqa al-tamām) ou encore "au-delà de la dernière limite» (wara'a al-ḡāya ${ }^{63}$. On reconnaît ici le Principe - et encore une fois l'inspiration est néoplatonicienne : le Premier n'est pas un tout (il n'a aucune possibilité et aucune partie, il est absolument nécessaire, incomposé et indivisible). Il doit donc être reconduit à un rang éminent par rapport aux autres êtres, le rang supérieur et transcendant qui doit appartenir à ce qui non seulement est, mais aussi donne l'être. Tout en n'ayant ni possibilité ni parties, le Principe possède idéalement non seulement tout ce dont il a besoin, mais aussi tout ce dont la totalité des êtres a besoin ${ }^{64}$.

62. Ilāh., IV, 3, p. 189, 5-6.

63. Ilāh., IV, 3, p. 186, 15 .

64. En Ilāh. IV, 3, p. 188, 3 et p. 189, 18, Avicenne distingue les significations de "plus que complet» (ce qui correspond au Principe), «complet» (qui correspond au principe mais 
J'ai mentionné la possible (non-)occurrence du terme totalitas à propos du Nécessairement Existant. En Ilāhiyyāt IV, 3, Avicenne établit des correspondances précises entre le tout et la complétude pour en conclure que le Principe ne peut pas être dit complet ou parfait (tāmm) précisément parce qu'Il excède le tout. En effet, si par "complet», on entend ce qui a tout ce qu'il faut à son être ${ }^{65}$, le Principe dont l'être déborde et qui est au-delà de la quiddité ne peut pas être considéré comme "complet» (il n'est en ce sens pas une totalité $\left.{ }^{66}\right)$. Il est au-dessus de la complétude. C'est à partir de son existence - en soi et non pas en vertu de quelque chose d'autre - que l'existence efflue pour se répandre sur les choses qui sont toutes. Si donc le Principe est "plus que complet », c'est qu'Avicenne distingue ce qui est entier et «en soi» de ce qui est tout (et a ou non des parties) ${ }^{67}$. Néanmoins, ce qui est plus qu'un tout ne peut pas être conçu en dehors de la relation avec ce qui est autre que lui, car ce qui est plus que complet est bien le Principe en tant qu'origine du flux ${ }^{68}$. Du point de vue théorique, la totalité

aussi à l'intelligence), «suffisant» (qui définit l'Âme du tout), « déficient» (qui qualifie tout ce qui est dans le monde sublunaire), "partie» (à la fois dans un sens général et dans le sens de dénombrable), «tout » et «ensemble». Il essaie également de retracer l'histoire des différents termes. C'est à Aristote qu'il se réfère (Métaphysique, Delta, 16, 25 et 26), ce qui est clair aussi par rapport à la terminologie. Cependant, tout le discours est inspiré par le thème néoplatonicien de ce qui est "plus que complet» ou «au-dessus de la complétude». Le fondement du flux est en fait la sur-complétude du Premier à partir duquel toute existence qui n'est pas le Premier efflue en faveur des choses.Voir aussi Ilāh., VIII, 6, p. 355, 3-10, où le Premier est d'abord défini comme «complet» ou "parfait» et ensuite comme "plus que complet» ou "plus que parfait». Le passage est dans un contexte qui peut bien être rapproché au Livre du Bien pur: C. d'Ancona, "Avicenna and the Liber... ».

65. Aristote, Métaphysique, Delta 26, 1023 b 25: « un tout se dit de ce à quoi ne manque aucune des parties qui sont dites constituer naturellement un tout»; et aussi Plotin, par Enn. III, 7 (45); VI, 4 (22), 5 (23); voir aussi la distinction chez Proclus entre teleiotes et olotes. À ce sujet, chez Proclus: T. Kobusch, «Das Eine ist nicht das Ganze. Die Idee der Totalität im Werk des Proklos, besonders in der Platonischen Theologie", in Proclus et la Théologie platonicienne (Actes du colloque international de Louvain, 13-16 mai 1998), A.-P. Segonds et C. Steel (dir.), Leuven - Paris, Leuven University Press, 2000, p. 311-323 (et en particulier p. 319 pour Avicenne). Des suggestions intéressantes à propos de la totalité chez Proclus: H. Dumery, «Proclus et la puissance de produire», in Néoplatonisme, Mélanges offerts à Jean Trouillard. Les Cahiers de Fontenay, n ${ }^{\circ}$ 19-22, mars 1981, p. 159-173.

66. Ilāh., IV, 3 .

67. Ilāh., IV ,3, p. 190, 1-191, 5. Au sens général ou au sens de ce qui peut être énuméré; Avicenne fait référence à la Métaphysique d'Aristote: Delta, 16, 25 et 26 mais aussi au néoplatonisme. Dans ce contexte, on trouve la mention de ce qui est un ensemble ( $\check{g} a m \bar{\imath})$, et donc un tout, dont les unités sont des membres. Cette idée est utilisée pour démontrer l'existence nécessaire du Principe; voir supra à propos de ğumla en tant que chaîne causale et Išärāt (IV, 9-15), où Avicenne parle de la série causale comme d'une totalité ou d'un ensemble qui doit donc nécessairement être close ou enfermée par un Principe.

68. Ilāh., III, 3, p. 344, 1-2, p. 343, 16 et p. 344, 5 ; ce qui semble une fois de plus se distancer de la conception plotinienne ou, plus exactement, l'interpréter: seules les quiddités sont, en tant qu'existantes, relatives. 
n'est pas pensable. Pour l'être vraiment, elle devrait inclure non seulement ce qui en parle, mais le discours qu'on en fait. Ou bien, pour être pensée, la totalité-une renvoie de façon inévitable à l'autre-multiple. D’ailleurs, si la création absolue est celle qu'instaurent la totalité des choses et les choses dans leur totalité, c'est qu'elle reflète ou redouble ce dont elle efflue ${ }^{69}$.

Olga L. Lizzini

Vrije Universiteit, Amsterdam

69. Plotin, Enn., VI, 4 (22), 2. Avicenne parle dans les termes du reflet ou du dessin de la connaissance parfaite de l'homme dont l'âme, l'intellect, devient «un monde intellectuel en acte» parallèle au «monde intelligible» de la quiddité du Tout. lāh., VIII, 7, p. 369, 17-370, 7 ; IX, 7, p. 425, 15-426, 4; 428, 10-11. 